\title{
Original artide \\ Profile of the glaucomas and intervention in a large eye care centre in South-East Nepal
}

\author{
Sarkar S1, Mardin C², Hennig A ${ }^{1}$ \\ ${ }^{1}$ Sagarmatha Choudhary Eye Hospital, Lahan, Siraha, Nepal \\ ${ }^{2}$ Department of Ophthalmology and University Eye Hospital, Friedrich-Alexander University Erlangen- \\ Nürnberg at Erlangen, Germany
}

\begin{abstract}
Introduction: Glaucoma is the second leading cause of blindness worldwide.

Objective:To estimate the prevalence and risk factors of glaucoma and modes of therapeutical intervention in a rural population of a developing country.

Patients and methods: A single centre-based cross-sectional study was carried out over a seven-month period in Sagarmatha Choudhary Eye Hospital (SCEH), Lahan/NEPAL, including the patients with glaucoma.

Outcome measurement: The patients' demography, type of the glaucomas, visual acuity, vertical cup-disc ratio, intra-ocular pressure and visual field findings were the parameters studied.

Results: A total of 3986 eyes with suspected glaucoma were identified. $53.7 \%$ of the patients were between 41 - 60 years of age. $53.9 \%$ were male. $26.5 \%$ were blind or severely visually impaired. $37.2 \%$ had IOP more than $35 \mathrm{mmHg}$. $46.4 \%$ had vertical cup-disc ratio (VCDR) higher than 0.8. Severe visual field defects were found in $28.4 \%$ eyes. 35.3\% eyes had primary angle-closure glaucoma, $22.6 \%$ eyes primary open-angle glaucoma, and $14.6 \%$ eyes normaltension glaucoma. $10.2 \%$ eyes suffered from lens induced glaucoma. Only in 9 eyes was pseudoexfoliation syndrome with glaucoma diagnosed. $25.7 \%$ eyes were advised for trabeculectomy. $11.5 \%$ eyes underwent extra-capsular cataract surgery. IOP lowering medication was prescribed in $5.9 \%$ patients.
\end{abstract}

Conclusions: The most common glaucoma seen in the out-patient department of a large eyecare centre in South-East Nepal is primary angle-closure glaucoma. $25 \%$ of the glaucoma patients are visually impaired or blind at the time of presentation. In more than one-fourth of the patients, trabeculectomy is the therapy of choice.

Keywords: glaucoma, South-East Nepal, North-East India, Bihar, blindness.

\section{Introduction}

Globally, in 2002, more than 161 million people were visually impaired, of whom 124 million had low vision and 37 million were blind. $27 \%$ of people with visual impairment were from South-East Asia. Glaucoma is

Received: 23.10.2009 Accepted: 02.12.2009

Correspondence and reprint request to: Dr. Albrecht Hennig, MD,

Sagarmatha Choudhary Eye Hospital,

Lahan, Siraha, Nepal.

E-mail: albrecht.hennig@erec-p.org the second leading cause of blindness worldwide (WHO, 2004). In India, it is the second leading cause of preventable blindness and the leading cause of irreversible blindness (Vijaya et al 2006). It is estimated that approximately 12 million Indians will be affected by the year 2010. Due to the increase and aging of the population, 16 million Indians will be affected due to glaucoma by the year 2020(Quigley \& Broman, 2006). There are some population studies on glaucoma in India. 
Most of them are from South-India (Dandona et al 2000a, Dandona et al 2000b, Ramakrishnan et al 2003, Vijaya et al 2005, Vijaya et al 2006, Vijaya et al 2008a, Vijaya et al 2008b, Thomas et al 2003). One study is from central India (Palimkar et al 2008). From NorthIndia, only one centre-based retrospective study has been reported (Das et al 2001). But there is no reported study on the prevalence of glaucoma from South-East Nepal and its adjacent North-East Indian states (Bihar, Uttar Pradesh, Bengal and Assam).

The aim of this study was to estimate the prevalence and risk factors of glaucoma and modes of therapeutical intervention in a rural population of a developing country, seen in a large ophthalmological referral centre, the Sagarmatha Choudhary Eye Hospital (SCEH), Lahan in South-East Nepal.

\section{Patients and methods}

In a seven-month period from mid-April to midNovember 2007, a total of 58,150 patients were screened and examined in the OPD by ophthalmologists or ophthalmic assistants. Out of them, 2009 (3.5 \%) patients with suspected glaucoma were referred to the glaucoma clinic.

The examinations were performed in two stages. First, distant and near visual acuity (with current refractive correction if any) were measured with Snellen's chart. Then pulse air tonometry (Keeler Inc., Great Britain) was determined in all eyes screened in the OPD. After the determination of visual acuity and pulse air tonometry, patients were screened for any ocular morbidity in the OPD. External eye examination, pupillary reaction and anterior segment examination were performed with torch light in a screening mode. The fundus was examined with direct ophthalmoscope.

The criteria for referral to the glaucoma clinic included: intraocular pressure (IOP) > $21 \mathrm{mmHg}$, optic nerve head changes suggestive of glaucoma (vertical cup-to-disc ratio [VCDR] $>0.3$, focal notching of cup, cups not obeying ISNT rule, deepening of the cup, thinning of the neuroretinal rim, laminar dot sign, over pass cupping, saucerisation of the cup, asymmetrical cupping between two eyes $>0.2$ etc.), suspected shallow anterior chamber found with or without history of headache, eye ache, coloured halos, family history of glaucoma and history of anti-glaucoma medication.
In the glaucoma clinic, at first IOP was again measured with Goldmann applanation tonometer. The mean of three consecutive readings was used for analysis. Gonioscopy was attempted in all participants with a Goldmann two mirror gonioscope (Haag Streit Inc., Switzerland) and the angles were graded according to RPC classification (Gupta et al 1975, Thomas et al 1996, Sihota et al 1998). If the posterior pigmented trabecular meshwork was not visible in the three quarters or more of the angle circumference in the primary position without manipulation in the presence of low illumination, the angle was considered occludable, otherwise it was open (i.e. grade 3 or less). If the patient was not cooperative with gonioscopy, the van Herrick technique was used to grade peripheral anterior chamber depth. When peripheral chamber was equal to or less than $14^{\text {th }}$ of corneal thickness, the angle was considered occludable. All participants with open angles determined on gonioscopy had their eyes dilated using tropicamide $1 \%$ and/or phenylnephrine $10 \%$. Participants who had dilatation deferred because of occludable/narrow angles had dilated examination performed after laser iridotomy either on the same day or on a subsequent day. Stereoscopic examination of the optic disc and parapapillary area was performed at the slit lamp using a 90-diopter lens and examination of the whole fundus with 20-diopter lens. VCDR was measured and recorded. Asymmetry of discs, notching, bayoneting, disc haemorrhages, peripapillary atrophy, tilted discs, etc were noted and recorded. The width and locations of the thinnest neuroretinal rim was also noted. Diurnal variation of IOP with pulse air tonometry was performed in patients with open angles with high IOP recorded on two separate occasions or only with disc changes suggestive of glaucoma. An absolute value of more than $30 \mathrm{mmHg}$ or a difference of more than 8 mmHg between the maximum and minimum values of IOP was taken as significant.

Pseudo-exfoliation (PEX) deposits on the corneal endothelium, iris and iris margin were examined using 20 times magnification before dilating the pupil. The anterior lens surface was also screened for any PEX material. PEX syndrome was diagnosed by the typical white deposits on the iris and/or anterior lens surface, uvea, anterior vitreous face, posterior capsule and even on the intraocular lens.

Cooperative patients during previous examinations of 
Goldmann's tonometry and gonioscopy underwent frequency doubling perimetry (FDT), (C-20-5 screening protocol). FDT score was determined by clinical scoring algorithm of FDT (Sponsel et al 1998). In case the findings were abnormal (defined as one area of abnormal sensitivity) or unreliable (defined as over 33 $\%$ of false positive and negative errors and fixation losses), the test was repeated after a short break with additional instructions.

In this study, the following criteria were used to diagnose an optic nerve head as glaucomatous: VCDR $>0.7$, focal notching of cup violating ISNT rule, asymmetry of cupping $>0.2$ between two eyes, disc changes such as thinning of neuroretinal rim, laminar dot sign, overpass cupping and saucerization of cup.

Criteria used for diagnosing glaucoma in this study were:

1. IOP $>21 \mathrm{mmHg}$ with glaucomatous optic nerve head with or without visual field defect in FDT.

2. Diurnal variation of IOP $>8 \mathrm{mmHg}$ along with glaucomatous optic nerve head with or without FDT defects.

3. Progressed glaucomatous optic nerve head with or without field defect found in FDT with IOP $<21$ mmHg.

4. Occludable angle with PAS on gonioscopy.

5. Closed angle found on gonioscopy.

The criteria used for various subtypes of glaucoma are described as follows:

Primary angle closure glaucoma (PACG) was classified into acute, intermittent, latent and chronic types. Patients with shallow anterior chamber depth with a functional block between the pupillary part of the iris and anterior lens surface or patients with a zipper- like closure of the angles and relatively normal anterior chamber depth comprised acute, intermittent and chronic types depending on clinical presentation. Asymptomatic patients with impending angle occlusion were grouped as latent angle closure.

Patients younger than 40 years with similar phenotype to primary open angle glaucoma (POAG) were labelled as juvenile open angle glaucoma (JOAG).

Ocular hypertensives (OHTN) were defined as a subset of patients with open angles, raised IOP without any optic nerve head change or visual field change.
Normal tension glaucoma (NTG) was diagnosed in patients with open angles and progressive glaucomatous optic nerve head changes with or without visual field changes in FDT in absence of raised IOP $>21 \mathrm{mmHg}$.

Glaucoma suspects subtypes included patients with open angles, IOP less than or equal to $21 \mathrm{mmHg}$ in the absence of any FDT changes with optic nerve head changes and patients with a strong family history of glaucoma in absence of optic nerve head changes or elevated IOP (greater than or equal to $22 \mathrm{mmHg}$ ).

Congenital glaucoma was diagnosed in patients with raised IOP due to immature trabecular meshwork with or without developmental anomalies of the eye present at birth.

Secondary glaucoma in patients was defined as increased IOP or glaucomatous optic nerve head changes with any ocular or systemic diseases predisposing to glaucoma.

Lens induced glaucoma (LIG) was diagnosed in patients with phacolytic, phacomorphic, phacotopic and lens particle induced glaucoma.

Absolute glaucoma was diagnosed in patients with endstage glaucoma without adequate evidence regarding the primary insult or cause contributing to glaucomatous optic nerve damage.

Patients with the following criteria were advised for anti-glaucoma medication under Lahan circumstances.

1. Eyes with VCDR $>0.8$ or glaucomatous optic nerve head changes with IOP 21-30 mmHg with or without FDT defect.

2. Eyes with suspected OHTN with IOP $>21 \mathrm{mmHg}$.

3. Eyes with suspected NTG with diurnal variation in IOP $<8$ mmHg with or without FDT defect.

The following patients were advised for Nd-YAG peripheral iridotomy.

1. Patients with intermittent angle closure glaucoma.

2. Fellow eyes of acute angle closure glaucoma.

3. Eyes with primary chronic angle closure glaucoma with cupping $<0.8$ and IOP $<35 \mathrm{mmHg}$.

4. Eyes with occludable angles as patients' follow-up was not reliable.

Surgical peripheral iridotomy was advised where Nd- 
YAG iridotomy could not be done due to very thick iris or blocked Nd-YAG iridotomy.

Trabeculectomy or combined surgery (trabeculectomy with SICS Fishhook Technique) were advised in the following eyes:

1. Eyes with VCDR equal to or greater than 0.8 or definitive glaucomatous optic nerve head with presenting IOP equal to or greater than $35 \mathrm{mmHg}$.

2. Eyes with PACG with failed peripheral iridotomy with IOP $>30 \mathrm{~mm}$ of $\mathrm{Hg}$.

3. Patients with VCDR $<0.8$ and IOP between 30-35 $\mathrm{mmHg}$ with field defect in FDT in one eye and other eye lost definitively due to glaucoma were advised for trabeculectomy in the better eye.

4. Eyes with the above criteria and significant cataract were intervened with combined surgery.

In eyes with congenital glaucoma, a combination of trabeculectomy plus trabeculotomy with Mitomycin C was envisaged. In patients with JOAG trabeculectomy with Mitomycin C was performed. For painful absolute glaucoma eyes trans-scleral cyclo-photocoagulation was advised.

\section{Results}

\section{Demographic data}

1615 (80.4 \%) patients came from Northern-India, whereas 394 (19.6 \%) came from Nepal. 1079 (53.7 \%) patients were aged between 41-60, 560 (27.9 \%) had age above 61 years and only 370 (18.4\%) patients were aged <41 years (Fig-1). 1082 (53.9\%) patients were male and 927 (46.1\%) female.

Figure-1

\section{Demographic data of patient age at the time of} presentation

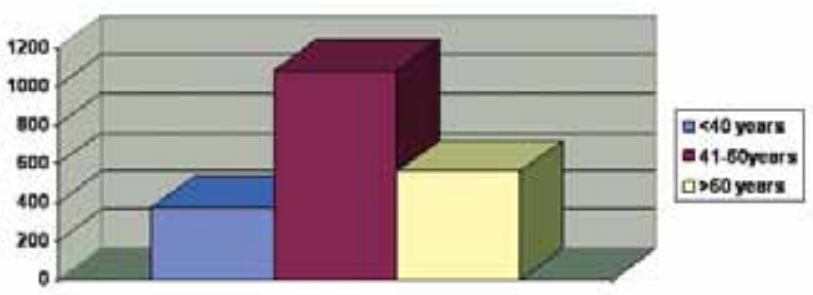

\section{Symptoms at presentation}

Painless dimness of vision was the main complaint in 1177 patients. 576 patients had dimness of vision with pain. 151 patients complained of headache and eye ache. 63 patients were complaining of irritation, watering etc. and found to have suspected glaucoma i.e., were detected accidentally. Halos were the main complaint in 42 patients.

\section{Visual status}

According to the 10th Revision of the WHO International Statistical Classification of Diseases, Injuries and Causes of Death, 289 (14.4\%) were blind at presentation and $243(12.1 \%)$ patients were severely visually impaired (SVI). 918 (45.7\%) patients had normal vision, while 559 (27.8\%) had visual impairment (VI). Among these 2009 patients, 483 eyes did not perceive any light (Fig-2).

Figure-2

Visual status at the time of presentation. Normal, visually impaired (VI), severely visually impaired (SVI), blind

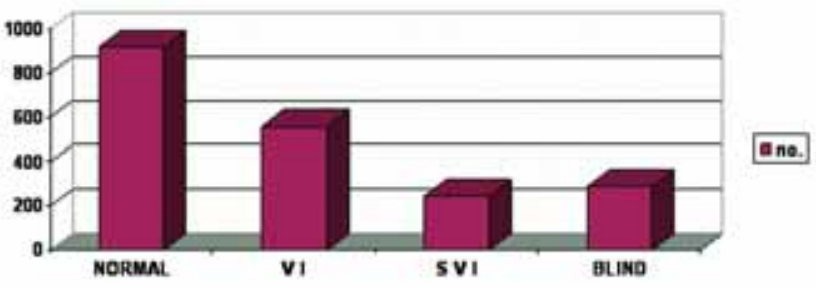

Distribution of intraocular pressure (IOP)

Out of 2009 patients, intraocular pressure could be determined in 3949 eyes. 1470 (37.2 \%) eyes had IOP $>35$ mmHg. 1169 (29.6 \%) eyes had IOP equal to or less than $21 \mathrm{mmHg}$, while 1310 (33.2 \%) eyes had IOP of $22 \mathrm{~mm}-35 \mathrm{mmHg}$. 21 eyes were phthisical and in 37 eyes IOP could not be determined.

Distribution of vertical cup disc ratio (VCDR) VCDR could be determined in 3630 eyes. 1684 (46.4 $\%)$ eyes had a VCDR >0.8; 848 (23.4\%) eyes were with VCDR 0.6-0.8 and 1098 (30 \%) eyes had VCDR $<0.6$. In 356 eyes, disc morphology could not be assessed.

\section{Frequency doubling testing}

Only in 664 eyes could reliable FDT findings be determined. 245 (36.9\%) eyes had normal FDT results, whereas 189 (28.5\%) eyes had severe defects. 160 (24.1\%) eyes and 70 eyes (10.5\%) had mild and moderate defects respectively. 


\section{Subtypes of glaucoma patients}

Based on the above-mentioned criteria, the different primary glaucoma subtypes are shown in Fig-3. Primary angle closure glaucoma was the most common subtype. The details of the subtypes are given in Fig-3.

Figure-3

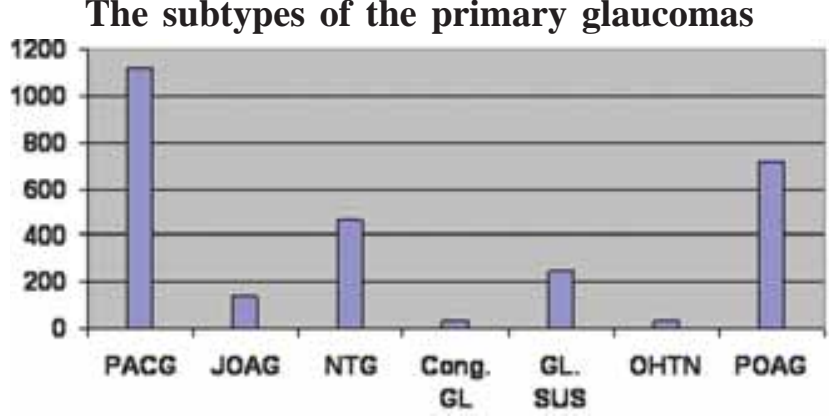

Primary angle closure glaucoma (PACG), juvenile openangle glaucoma (JOAG), normal-tension glaucoma (NTG), congenital glaucoma (Cong. GL), glaucoma suspect, ocular hypertension (OHTN), primary openangle glaucoma (POAG).

\section{Primary angle closure glaucoma (PACG)}

Out of 2009 patients, 3166 eyes were diagnosed to have glaucoma. Among these, 1120 (35.3 \%) were cases with PACG and comprised the largest group. The most common subtype of PACG was chronic PACG (977/ 1120; $87.2 \%) .87$ eyes (87/1120; $7.8 \%$ ) had a latent angle closure. 45 eyes (45/1120; $4 \%)$ had intermittent angle closure and 11 eyes (11/1120; $1 \%$ ) had acute attack of angle closure.

Primary open angle glaucoma (POAG) was diagnosed in 714 eyes (714/ 3166; 22.6 \%). 142 (142/3166; 4.5 $\%)$ eyes were diagnosed to have juvenile open angle glaucoma (JOAG). 461 (461/3166; $14.6 \%$ ) eyes had NTG. Only 33 (1\%) eyes had congenital glaucoma. 249 (249/3166; $7.9 \%)$ eyes were diagnosed as glaucoma suspects. Only 14 eyes were diagnosed to have ocular hypertension (OHT).

\section{Secondary glaucomas}

412 (412/3166; $13 \%)$ eyes were diagnosed to have secondary glaucoma. LIG was the most common cause in this group (324/3166; $10.2 \%)$. Only 9 eyes were diagnosed to have pseudoexfoliation syndrome. 37(1 $\%)$ eyes had neovascular glaucoma. 5 eyes had glaucoma due to angle recession. Uveitic glaucoma was found in 14 eyes. Only 2 eyes were diagnosed to have pigmentary glaucoma. 16 eyes had aphakic glaucoma.
Figure-4

Subtypes of secondary glaucomas

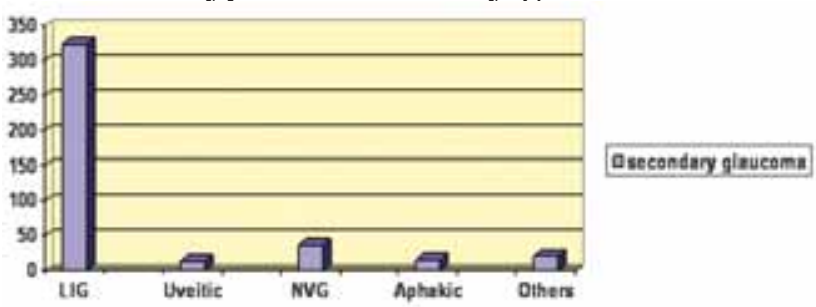

Only 5 eyes had steroid induced glaucoma (Fig-4).

Lens induced glaucoma (LIG), uveitic glaucoma (UG), neovascular glaucoma (NVG), aphakic glaucoma (Aphakic) and others

\section{Therapeutic intervention}

Out of 3986 eyes of 2009 patients, 1024 (25.7 \%) were advised for trabeculectomy. Nd-YAG peripheral iridotomy was advised in 245 (6.1\%) eyes. 41 (1\%) eyes were referred for surgical peripheral iridotomy. Trans-scleral cyclo-photocoagulation was performed in 89 (2.2\%) eyes. Combined surgery was advised in 149 (3.7 \%) eyes. 458 (11.5 \%) eyes were referred for extracapsular cataract surgery (ECCE) with posterior chamber intraocular lens (PCIOL). Antiglaucoma medication was applied only in 236 (5.9\%) eyes. 134 patients were asked for follow-up without therapy.

\section{Discussion}

Among the glaucomas we found that PACG was the most common subtype (36.7\%), which is very similar to the findings of another centre-based study from North-India (36.6\%) (Das et al 2001). One study from North-India confirms our finding that chronic PACG is the most common subtype of PACG (Sihota et al 1998).

A remarkably high number of eyes with NTG (14.6\%) was diagnosed in our study compared to the report $(0.62$ \%) by Das et al (2001). Here, a drawback of this study may become obvious, as no phasing could be done regularly in this setting. So, elevated IOP values may have been missed and a high false positive number of NTG may have been found. A very low number of PEX glaucoma was noted, which is similar to the literature (Thomas et al 2003, Das et al 2001) in the Indian subcontinent. This is in contrast to other parts of the world where secondary glaucoma due to PEX accounts for over $25 \%$ of all glaucoma (SchlötzerSchrehardt et al 2002). 
Among secondary glaucomas, we found LIG to be the largest group (324 eyes; $81 \%$ ). Poverty, absence of good quality and affordable ophthalmic services, ignorance about the disease and illiteracy prevent patients from cataract surgery at an early stage. The next largest groups were neovascular glaucoma (37 eyes; 9 \%) due to Eale's disease, diabetes and others. Aphakic glaucoma was found to be only $4 \%$ in comparison to previous findings reported by Agrawal et al (1982). As ECCE with PCIOL implantation has now become the standard cataract surgical technique, this type of glaucoma is diminishing.

Recently, frequency doubling perimetry has emerged as a valuable tool for detecting glaucoma (Iwase et al 2007, Mansberger et al 2005). In those studies, reliable FDT results could be found in $93 \%-98 \%$ eyes. But in comparison, we could get reliable FDT results in only 664 (664/3986; 16.7 \%) eyes. Most of the participants did not have any experience with handling electronical devices or even sitting on a chair. In a patient population like ours, determining the visual field loss even with FDT turned out to be very difficult. Therefore, to diagnose glaucoma one has to depend mainly on clinical assessment and examinations like accurate applanation tonometry, gonioscopy in all cases and stereoscopic disc examination.

Patients usually come to SCEH after travelling for 2-3 days from the remotest parts of Nepal and North-East India. Most of them are farmers or agriculture labourers. Therefore the patient numbers in the OPD of SCEH vary on farming seasons and weather. In winter, OPD patients rise to 600 per day. They often plan and save their money for 6-12 months to come for treatment and they face various barriers for regular follow-up. These are poverty, remoteness of their villages, seasonal floods (June-September), harvesting seasons and an unstable and unpredictable political situation in the eastern Tarai of Nepal and in northern India. Because of the geopolitical situation of the region and socioeconomic status, patients usually come with eye diseases at an advanced stage. Poor compliance in the use of medicines and follow-up visits requires a special management plan for patients with early and suspected glaucoma to prevent blindness.

Most of the latest anti-glaucoma drugs are now available in urban areas in Nepal and India, but not for the rural population. Especially for rural and poor patients, the medicine is neither available nor affordable. The only option would be timolol and pilocarpine. However, our experience with medical treatment showed that only $20 \%$ of the patients came for the next follow-up after starting drugs, out of which $40 \%$ had already stopped medication by themselves. Due to uncertain follow-up by the patients, non-compliance to anti-glaucoma medication and absence of easy availability of drugs in the villages, the authors think that it is better to advise early surgical treatment where indicated.

According to a study, only $22 \%$ of PAC suspects may progress to PAC, but none to PACG (Thomas et al 2003). But considering the circumstances in Lahan, the authors think it is better to advise prophylactic peripheral iridotomy or iridectomy for patients with occludable angles.

Similar to the literature(Das et al 2001), a high proportion of blindness due to advanced glaucoma at presentation was found in our study. Almost $1 / 4^{\text {th }}$ (26 $\%)$ of the patients were severely visually impaired or blind at the time of presentation. This indicates an urgent need for early detection and treatment of glaucoma patients in this region.

\section{Conclusion}

The most common glaucoma seen in the out-patient department of a large eye-care centre in South-East Nepal is primary angle-closure glaucoma. $25 \%$ of the glaucoma patients are visually impaired or blind at the time of presentation. In more than one fourth of the patients, trabeculectomy is the therapy of choice.

We have attempted to define the profile of glaucoma on patients from remote parts of Nepal and North-India. Due to the high volume of patients, we may have missed patients with early glaucoma during our screening in the OPD, which may have caused a referral bias in this study. Although this is a single centre-based study, it could help to understand the severity of glaucoma, the profile of its different subtypes and ways of intervention in this region. It may also provide useful background information for planning population- based glaucoma studies and strategies to fight blindness due to glaucoma in this region.

\section{References}

Agarwal HC, Sood NN, Kalra BR et al (1982). Secondary glaucoma. Indian J Ophthalmol;30:121-24. 
Dandona L, Dandona R, Mandal P, Srinivas M, John RK, McCarty CA, et al (2000a). Angle-closure glaucoma in an urban population in southern India: The Andhra Pradesh eye disease study. Ophthalmology;107:1710-6.

Dandona L, Dandona R, Srinivas M, Mandal P, John RK, McCarty CA, et al (2000b). Open-angle glaucoma in an urban population in southern India: The Andhra Pradesh eye disease study. Ophthalmology;107:1702-9.

Das J, Bhomaj S, Chaudhuri Z, Sharma P, Negi A, Dasgupta A (2001). Profile of glaucoma in a major eye hospital in north India.Indian J Ophthalmol.;49(1):25-30.

Gupta S, Sood NN, Dayal Y (1975). Angle closure glaucoma IV. Eastern Arch Ophthalmol;3:238-42.

Iwase A, Tomidokoro A, Araie M, Shirato S, Shimizu H, Kitazawa Y; Tajimi Study Group (2007). Performance of frequency-doubling technology perimetry in a population-based prevalence survey of glaucoma: the Tajimi study. Ophthalmology.;114(1):27-32. Epub 2006 Oct 27.

Mansberger SL, Johnson CA, Cioffi GA, Choi D, Krishnadas SR, Srinivasan M, Balamurugan V, Kim U, Smith SD, Wilkins JH, Gritz DC (2005). Predictive value of frequency doubling technology perimetry for detecting glaucoma in a developing country.J Glaucoma. (2):128-34.

Palimkar A Khandekar R, Venkataraman V (2008). Prevalence and distribution of glaucoma in central India (Glaucoma Survey - 2001).Indian J Ophthalmol.;56(1):57-62.

Quigley HA, Broman AT (2006). The number of people with glaucoma worldwide in 2010 and 2020. Br J Ophthalmol;90:262-7.

Ramakrishnan R, Nirmalan PK, Krishnadas R, Thulasiraj RD, Tielsch JM, Katz J et al (2003). Glaucoma in a rural population of southern India: The Aravind Comprehensive Eye Survey. Ophthalmology;110:1484-90.

Schlötzer-Schrehardt U, Küchle M, Jünemann A, Naumann GOH (2002). Relevance of the pseudoexfoliation syndrome for the glaucomas. Ophthalmologe. (9):683-90.
Sihota R, Agarwal HC (1998). Profile of the subtypes of angle closure glaucoma in a tertiary hospital in north India.Indian J Ophthalmol.;46(1):25-9.

Sponsel WE,Arango S, Trigo Y, Mensah J (1998). Clinical classification of glaucomatous visual field loss by frequency doubling perimetry. Am J Ophthalmol.125(6); 830-36.

Thomas R, George R, Parikh R, Muliyil J, Jacob A (2003). Five year risk of progression of primary angle closure suspects to primary angle closure: a population based study. Br J Ophthalmol. (4):4504.

Thomas R, George T, Braganza A, Muliyil J (1996). The flashlight test and Van Herick's test are poor predictors for occludable angles. Aust NZ J Ophthalmol;24:251-56.

Thomas R, Paul P, Muliyil (2003). Glaucoma in India. J Glaucoma;12 (1):81-7.

Vijaya L, George R, Arvind H, Baskaran M, Paul PG, Ramesh SV et al (2006). Prevalence of angleclosure disease in a rural southern Indian population. Arch Ophthalmol;124:403-9.

Vijaya L, George R, Arvind H, Baskaran M, Raju P, Ramesh SV et al (2006). Prevalence and causes of blindness in the rural population of the Chennai Glaucoma Study. Br J Ophthalmol;90:407-10.

Vijaya L, George R, Arvind H, Baskaran M, Ve Ramesh S, Raju P et al (2008b). Prevalence of Primary Angle-Closure Disease in an Urban South Indian Population and Comparison with a Rural Population The Chennai Glaucoma Study. Ophthalmolgy; 115 (4): 655-660.

Vijaya L, George R, Baskaran M, Arvind H, Raju P, Ramesh SV et al (2008a). Prevalence of Primary Open-angle Glaucoma in an Urban South Indian Population and Comparison with a Rural Population The Chennai Glaucoma Study. Ophthalmology; 115 (4): 648-654.

Vijaya L, George R, Paul PG, Baskaran M, Arvind H, Raju P et al (2005). Prevalence of open-angle glaucoma in a rural south Indian population. Invest Ophthalmol Vis Sci;46:4461-7.

WHO Fact Sheet No 282 (2004).

Source of support: nil. Conflict of interest: none 